\title{
Universality in Distribution of Monogamy Scores for Random Multiqubit Pure States
}

\author{
Soorya Rethinasamy ${ }^{1,2}$, Saptarshi Roy ${ }^{1}$, Titas Chanda ${ }^{1,3}$, Aditi Sen(De $)^{1}$, Ujjwal Sen ${ }^{1}$ \\ ${ }^{1}$ Harish-Chandra Research Institute, HBNI, Chhatnag Road, Jhunsi, Allahabad 211 019, India \\ ${ }^{2}$ Birla Institute of Technology and Science, Pilani, Rajasthan 333031, India and \\ 3 Instytut Fizyki im. Mariana Smoluchowskiego, \\ Uniwersytet Jagielloński, Łojasiewicza 11, 30-348 Kraków, Poland.
}

\begin{abstract}
Monogamy of quantum correlations provides a way to study restrictions on their sharability in multiparty systems. We find the critical exponent of these measures, above which randomly generated multiparty pure states satisfy the usual monogamy relation, and show that the critical power decreases with the increase in the number of parties. For three-qubit pure states, we detect that W-class states are more prone to being nonmonogamous as compared to the GHZ-class states. We also observe a different criticality in monogamy power up to which random pure states remain nonmonogamous. We prove that the "average monogamy" score asymptotically approaches its maximal value on increasing the number of parties. Analyzing the monogamy scores of random three-, four-, five- and six-qubit pure states, we also report that almost all random pure six-qubit states possess maximal monogamy score, which we confirm by evaluating statistical quantities like mean, variance and skewness of the distributions. In particular, with the variation of number of qubits, means of the distributions of monogamy scores for random pure states approach to unity which is the algebraic maximum - thereby conforming to the known results of random states having maximal multipartite entanglement in terms of geometric measures.
\end{abstract}

\section{INTRODUCION}

Quantum entanglement [1], one of the most striking features in quantum mechanics, is the essential resource [2] for a plethora of quantum information protocols like quantum teleportation [3], quantum dense coding [4], entanglement-based quantum cryptography [5], one-way quantum computation [6] etc. These protocols revolutionize the existing communication and computation schemes based on laws of classical mechanics. Due to immense importance of entanglement, over the years, several detection methods like partial transposition [7] based on positive maps [8], entanglement witness, [911], and quantifiers such as distillable entanglement [12], entanglement of formation [13, 14], logarithmic negativity $[15,16]$ have been proposed. On the other hand, it has also been realized that quantum mechanical systems can exhibit nonclassical phenomena which cannot be explained by using the theory of entanglement, and hence a different resource theory has been developed where unlike separable states, "classically correlated" states in the computational basis are the free states [17]. These measures, independent of entanglement, belonging to a finegrained paradigm of quantum correlations (QC), have their origin in the concepts of information theory or geometry of states or thermodynamics. Examples of such measures include quantum discord [18], geometric quantum discord [19], quantum work deficit [20, 21], and quantum deficit [22] (see review [17]).

Beyond the bipartite domain, understanding of $\mathrm{QC}$, even for pure states shared by multiple parties, is limited due to its complex structure. In this paper, we study the distribution of QC among the various parties of a random multipartite quantum state, with the help of the concept of "monogamy" [23]. Unlike classical correlations, which can be shared freely between different parties, monogamy of QC [23-25] restrains arbitrary sharing of $\mathrm{QC}$ among the various parties of a multipartite quantum state. Specifically, in a tripartite scenario, if two parties share maximal QC, they cannot at all be quantum correlated with the third party. The quantitative version of this concept, in the form of an inequality, was formulated by Coffman, Kundu, and Wootters [25] involving bipartite QC measures for arbitrary states having varying number of parties. Apart from its fundamental importance [24, 26-31], such notion turned out to have applications in diverse areas of physics including quantum crypography [32, 33], identification of quantum channels [34], and many body physics [35-37]. The monogamy inequality can also give rise to a quantity, known as monogamy score [38], which can take both negative as well as non-negative values and can be used for characterization of QC. We ask here the following question: Do non-negative monogamy scores for random multiparty pure states behave in a fashion that is similar to the known results obtained for geometric measure of entanglement (cf. [39, 40])? We answer it affirmatively, for a large spectrum of bipartite QC measures, required to construct the monogamy score.

Most of the computable measures are known to be nonmonogamous for three-qubit pure states while their squares often satisfy the monogamy relations $[25,31,41-$ 45]. For a given QC, the power (exponent) that makes all three qubit pure states to be monogamous often lies between 1 and 2. An exception is quantum work deficit [26]. We first find out the critical power required for obtaining monogamy for all three-qubit pure states. We observe that criticalities are different for the two classes inequivalent with respect to stochastic local operations and classical communication (SLOCC)-viz. the GHZand the W-classes [46].

When the above analysis is repeated for randomly 
generated pure states of four-, five- and six-qubits, we find that the critical value in power of the bipartite QC measures in the monogamy score decreases with the increase in the number of parties. Moreover, we observe that for entanglement measures, the percentage of states to exhibit nonmonogamy jumps to a lower value when the powers of entanglement measures are strictly greater than 0 (upto numerical accuracy).

We finally show that the monogamy score approaches its algebraically allowed maximum value on increasing the number of qubits for random pure states irrespective of the QC measure. We then examine several statistical quantities like mean, variance and skewness of the distributions of monogamy scores for random pure states of three-, four-, five- and six-qubits. Specifically, such investigations reveal that there is a transition in the distribution of monogamy score from lower mean values to higher mean values, and from being right-skewed to left-skewed with the increase in the number of qubits from three to six. Therefore, we conclude that the monogamy scores based on any quantum correlation measure reaches their maximum value for most of the random pure states with moderate number of parties, thereby supporting an analytical result (in the asymptotic limit) obtained in this paper and mimicking the pattern of multiparty geometric measure of entanglement.

The paper is organized as follows. We discuss the concept of monogamy score in Sec. II. Subsequently, the criticalities in the power of bipartite measures with respect to their monogamy scores are investigated in Sec. III. A comparative study between the criticalities of the GHZ- and the W-class states are given in Sec. III A, followed by a similar analysis for random pure states with higher number of qubits in Sec. III B. We then shift our focus to the actual distributions of monogamy score in Sec. IV and extract universal features of these distributions by computing their statistical properties. We then study the difference in the distributions for the GHZ- and $\mathrm{W}$-class states in Sec. IV A and then move on to a higher number of parties in Sec. IV B. We present concluding remarks in Sec. V.

\section{MONOGAMY SCORES}

The monogamy score $[24,25,38]$, for an arbitrary $N$ party quantum state, $\rho_{12 \ldots N}$, with respect to a bipartite quantum correlation measure, $\mathcal{Q}$, with the first party [47] as the "nodal" observer, is defined as

$$
\delta_{\mathcal{Q}} \equiv \delta_{\mathcal{Q}}\left(\rho_{12 \ldots N}\right)=\mathcal{Q}_{1: \text { rest }}-\sum_{i=2}^{N} \mathcal{Q}_{1: i}
$$

where $\mathcal{Q}_{1 \text { :rest }} \equiv \mathcal{Q}\left(\rho_{1 \text { :rest }}\right)$ is the quantum correlation in the 1:rest bipartition and $\mathcal{Q}_{1: i} \equiv \mathcal{Q}\left(\rho_{1: i}\right)$ denotes the quantum correlation $\mathcal{Q}$ between the nodal party and the $i^{\text {th }}$ party of the reduced density matrix, $\rho_{1 i}$ $(i=2,3, \ldots, N)$ of $\rho_{12 \ldots N} . \delta_{\mathcal{Q}}\left(\rho_{12 \ldots N}\right) \geq 0$ implies that the state, $\rho_{12 \ldots N}$, is monogamous with respect to $\mathcal{Q}$, and otherwise it is nonmonogamous.

In this paper, the chosen measures of quantum correlation (QC) from the entanglement-separability paradigm are concurrence, entanglement of formation, negativity, and logarithmic negativity, while from the information theoretic genre, we opt for quantum discord and quantum work deficit. The measures are chosen due to their analytical or numerical computability. For definitions of the above measures, see Appendix A.

\section{CRITICALITIES IN MONOGAMY POWER}

It is known that there exist three- or more-party quantum states which violate the monogamy relation for certain QC measures. At the same time, it was proven that if one considers squares of several of these measures, such states become monogamous [25, 44, 45]. QC measures, exhibiting this behavior include all the computable measures considered in this paper except quantum workdeficit [26].

We address here the question whether instead of an integer power, QC measures raised to positive real numbers are enough to satisfy the monogamy relation for threequbit pure states and for states with a higher number of parties. Specifically, we consider

$$
\delta_{\mathcal{Q}^{\alpha}}=\mathcal{Q}_{1: \text { rest }}^{\alpha}-\sum_{i=2}^{N} \mathcal{Q}_{1: i}^{\alpha}
$$

for some positive real number, $\alpha$, which typically lies between 1 and 2. In this respect, one should note that if $\mathcal{Q}$ is a valid measure of $\mathrm{QC}, \mathcal{Q}^{\alpha}$, where $\alpha$ is a positive real number, is also a valid measure. Moreover, notice that if $\mathcal{Q}\left(\rho_{1: i}\right)<1$ and if $\mathcal{Q}\left(\rho_{1 \text { :rest }}\right)<1$, we have $\delta_{\mathcal{Q}^{\alpha \rightarrow \infty}} \rightarrow 0$ for arbitrary multiqubit states. It implies that in the asymptotic limit $(\alpha \rightarrow \infty)$, all states become monogamous. Therefore it is interesting to examine whether there exists a finite value of $\alpha=\alpha_{c}$, such that for all pure states $\delta_{\mathcal{Q}^{\alpha}} \geq 0$, for $\alpha \geq \alpha_{c}$. Let us call $\alpha_{c}$ as the critical monogamy power. Furthermore, for $\alpha \rightarrow 0$, it trivially follows that $\delta_{\mathcal{Q}^{\alpha \rightarrow 0}} \rightarrow-N+2$, for an $N$-party state, which reduces to -1 , for three-qubit states, implying violation of monogamy inequality for all states, independent of $\mathcal{Q}$. Let us now define a quantity which quantifies the number of Haar uniformly generated states, violating monogamy relation for a given $\mathcal{Q}$, among all multiparty random pure states. We call it as the fraction of nonmonogamous states, $f$. Mathematically, for a given $\mathcal{Q}$ and $\alpha$,

$$
f=\frac{\text { number of nonmonogamous states }}{\text { total number of randomly generated states }} \text {. }
$$

in the limit of a large total number of states generated. When $\alpha \rightarrow 0, f \rightarrow 1$ for all multiparty random pure states. 
Assuming continuity, one can expect that in the neighborhood of $\alpha=0, f$ remains close to unity. Interestingly, however, our analysis reveals that the fraction of nonmonogamous states, $f$, remain frozen to unity even for some finite values of $\alpha$ leading to another criticality of $\alpha$. We denote this critical value of $\alpha$ upto which the value of $f$ stays unity as $\alpha_{p}$.
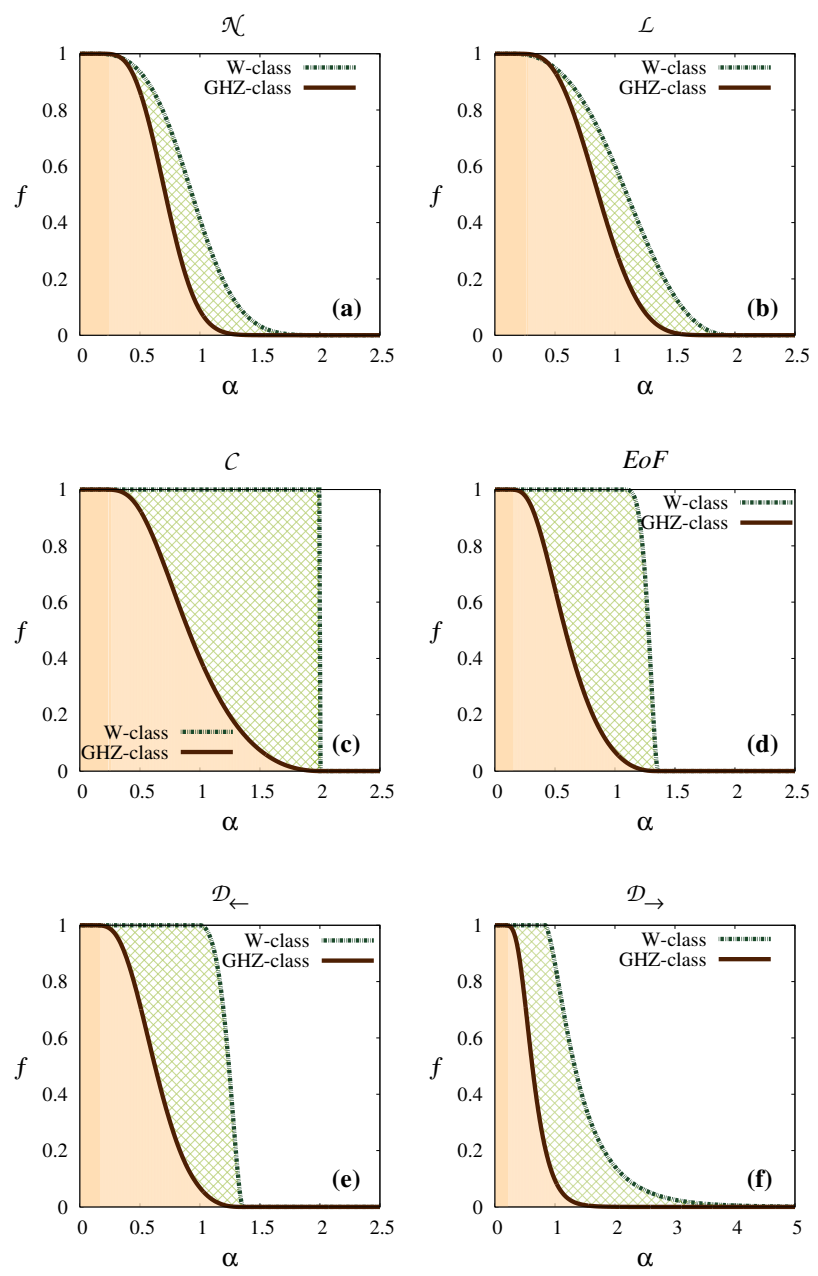

FIG. 1: (Color online.) Trends in fraction of nonmonogamous states, $f$, against monogamy power, $\alpha$, of QC measures for the GHZ- and the W-class states. The figures labelled (a)(f) show $f$, for negativity $(\mathcal{N})$, logarithmic negativity $(\mathcal{L})$, concurrence $(\mathcal{C})$, entanglement of formation $(E o F)$, quantum discord by measurement in the second party $\left(\mathcal{D}_{\leftarrow}\right)$ and in the first party $\left(\mathcal{D}_{\rightarrow}\right)$ of a quantum state. The shaded regions under each curve gives the area under the nonmonogamy fraction denoted as $\mathcal{M}$ (see Eq. (4)). All axes are dimensionless.

In this section, we investigate both the criticalities, $\alpha_{c}$ as well as $\alpha_{p}$, for both entanglement-based and information-theoretic measures of QC. Specifically:

1. We Haar uniformly generate $10^{6}$ three-qubit states [46] and perform a comparative study between the $\alpha_{p}$ and $\alpha_{c}$ values for different classes of three-qubit pure states.
2. To investigate the effect of the increase in the number of parties on $\alpha_{c}$, we also randomly generate multiqubit pure states having four, five, and six parties.

\section{A. Comparative study between GHZ- and W-class states}

The set of three-qubit pure states consists of two disjoint subsets belonging to two SLOCC inequivalent classes, namely the GHZ- and the W-class [46]. Note that the states from the $\mathrm{W}$-class is a set of measure zero and hence in the generation of random three-qubit pure states, W-class states cannot be found, thereby requiring an independent simulation for the $\mathrm{W}$-class states. We Haar uniformly generate three-qubit pure states randomly from both the classes and compare their respective $\alpha_{c}$ and $\alpha_{p}$ for both entanglement and informationtheoretic measures. Our analysis reveals that the states from the GHZ- and the W-class show qualitatively distinct behavior with respect to criticalities which are independent of the choice of the QC measures. We find (see Figs. 1 and 2, and Table. I :

1. For a large set of values of $\alpha$, the fraction of nonmonogamous states for the GHZ-class is less than that of the W-class, i.e., $f_{\mathcal{Q}^{\alpha}}^{\mathrm{GHZ}} \leq f_{\mathcal{Q}^{\alpha}}^{\mathrm{W}}$, for any quantum correlation measure $\mathcal{Q}$. It implies that W-class states are more nonmonogamous than the GHZclass states.

2. The value of $\alpha_{p}$ obtained for the GHZ-class, denoted by $\alpha_{p}^{\mathrm{GHZ}}$ is lower than that of the W-class, $\alpha_{p}^{\mathrm{W}}$, i.e., $\alpha_{p}^{\mathrm{GHZ}} \leq \alpha_{p}^{\mathrm{W}}$.

3. Like $\alpha_{p}$, the critical value of $\alpha$ for which all states become monogamous is always smaller for the GHZ-class states, $\alpha_{c}^{\mathrm{GHZ}} \leq \alpha_{c}^{\mathrm{W}}$, where $\alpha_{C}^{\mathrm{GHZ}}$ and $\alpha_{c}^{\mathrm{W}}$ denote critical values of $\alpha$ for which $\delta_{\mathcal{Q}^{\alpha}} \geq 0$ for the GHZ- and the W-class respectively. This result can be interpreted as the consequence of $f_{\mathcal{Q}^{\alpha}}^{\mathrm{GHZ}} \leq f_{\mathcal{Q}^{\alpha}}^{\mathrm{W}}$.

4. For concurrence, we know that $\delta_{\mathcal{C}^{2}}=0$ for all Wclass states. We find that $\alpha_{p}=\alpha_{c}=2$ for all states from the $\mathrm{W}$-class. It implies that all values of $\alpha<\alpha_{p}=\alpha_{c}=2$, we have $f=1$, i.e., all randomly chosen $\mathrm{W}$-class states are nonmonogamous with respect to $\mathcal{C}^{\alpha}$ (i.e., $\delta_{\mathcal{C}^{\alpha}}<0$ ) for $\alpha<2$. Moreover, $f$ has a finite jump from 1 to 0 at $\alpha=2$, which indicates the known result of vanishing three-tangle for the W-class states [46].

5. Interestingly, for the W-class states, $\alpha_{c}$ of $\mathcal{L}$ and $\mathcal{C}$ coincide, at $\alpha_{c}=2$, implying that no finite real values of $\alpha$ exist between 0 and 2 to make all states monogamous. Such characteristic is also seen for the states from the GHZ-class in case of monogamy relation of concurrence. 
6. We also observe that $\alpha_{p}$ is reasonably high for the W-class states in case of concurrence $(\mathcal{C})$, entanglement of formation $\left(E_{o F}\right)$, quantum discord $\left(\mathcal{D}_{\leftarrow}\right.$ and $\left.\mathcal{D}_{\rightarrow}\right)$, and work-deficit $\left(\mathcal{W}_{\leftarrow}\right.$ and $\left.\mathcal{W}_{\rightarrow}\right)$. In all these cases, $\alpha \gtrsim 1$.

The above picture remains qualitatively similar for both entanglement-based and information theoretic QC measures. However, there are some differences also. In particular:

1. For quantum work deficit, we find that, $\alpha_{c}>10$ independent of the party on which measurements are performed. To our knowledge, this is the only known measure of $\mathrm{QC}$ which requires such a high value of $\alpha$ to satisfy monogamy relation.

2. In case of information-theoretic measures, both $\alpha_{c}$ and $\alpha_{p}$ depend on the party where the measurement is performed. For example, in case of quantum discord, the value of $\alpha_{c}$ is drastically high when the measurement is carried out on the first party (see Table. I).

A detailed analysis of the critical monogamy power of random pure states will be important for arguing monogamy score as a valid multiparty QC measure from the perspective of the properties of random pure states.
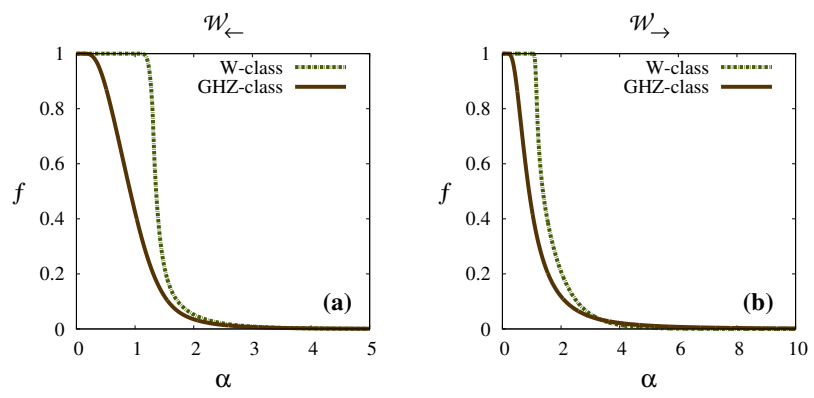

FIG. 2: (Color online.) Behavior of fraction of nonmonogamous states $(f)$ vs. monogamy power $(\alpha)$ for quantum work deficit when measurement is in (a) the second $\left(\mathcal{W}_{\leftarrow}\right)$ and (b) the first part $\left(\mathcal{W}_{\rightarrow}\right)$ of the $A: B C, A: B$, and $A: C$ bipartition of a given three-qubit pure state, $|\psi\rangle_{A B C}$. All axes are dimensionless.

Let us now introduce a measure to quantify the nonmonogamous nature of a given class of multipartite states with respect to a given $\mathrm{QC}$ measure, $\mathcal{Q}$ for all $\alpha \leq \alpha_{c}$. It is defined as the area under the curve of the fraction of nonmonogamous states with respect to the monogamy power of the considered quantum correlation measure $\mathcal{Q}$, which for a given class of states, reads as

$$
\mathcal{M}_{\mathcal{Q}}=\int_{0}^{\alpha_{c}} f_{\mathcal{Q}^{\alpha}} \mathrm{d} \alpha
$$

provided $\alpha_{c}$ is finite. See Table. II for $\mathcal{M}_{\mathcal{Q}}$ values for different QC measures. Note that $\mathcal{M}_{\mathcal{Q}}$ is a single number which composes information of both $\alpha_{c}$ and $\alpha_{p}$. As

\begin{tabular}{|c|c|c|c|}
\hline Measure & Class & $\alpha_{p}$ & $\alpha_{c}$ \\
\hline \multirow{2}{*}{ Negativity $(\mathcal{N})$} & GHZ & 0.1467 & 1.6735 \\
\cline { 2 - 4 } & $\mathrm{W}$ & 0.0991 & 1.9885 \\
\hline \multirow{2}{*}{ Logarithmic negativity $(\mathcal{L})$} & GHZ & 0.1497 & 1.8540 \\
\cline { 2 - 4 } & $\mathrm{W}$ & 0.0991 & 2 \\
\hline \multirow{2}{*}{ Concurrence $(\mathcal{C})$} & GHZ & 0.1470 & 2 \\
\cline { 2 - 4 } & $\mathrm{W}$ & 2 & 2 \\
\hline \multirow{2}{*}{ Entanglement of formation $(E o F)$} & GHZ & 0.0866 & 1.3520 \\
\cline { 2 - 4 } & $\mathrm{W}$ & 1.0410 & 1.4280 \\
\hline \multirow{2}{*}{ Quantum discord $($ left $)(\mathcal{D} \rightarrow)$} & $\mathrm{GHZ}$ & 0.1163 & 3.4317 \\
\cline { 2 - 4 } & $\mathrm{W}$ & 0.8382 & 9.3492 \\
\hline \multirow{2}{*}{ Quantum discord (right) $\left(\mathcal{D}_{\leftarrow}\right)$} & $\mathrm{GHZ}$ & 0.0968 & 1.3520 \\
\cline { 2 - 4 } & $\mathrm{W}$ & 0.9797 & 1.3608 \\
\hline \multirow{2}{*}{ Work deficit (left) $\left(\mathcal{W}_{\rightarrow}\right)$} & $\mathrm{GHZ}$ & 0.1183 & $>10$ \\
\cline { 2 - 4 } & $\mathrm{W}$ & 0.9630 & $>10$ \\
\hline \multirow{2}{*}{ Work deficit (right) $\left(\mathcal{W}_{\leftarrow}\right)$} & $\mathrm{GHZ}$ & 0.0989 & $>10$ \\
\cline { 2 - 4 } & $\mathrm{W}$ & 0.9799 & $>10$ \\
\hline
\end{tabular}

TABLE I: Comparative study of values of the two criticalities, $\alpha_{p}$ and $\alpha_{c}$ (see main text) for the GHZ- and the W-class states.

already argued before, $\mathcal{M}_{\mathcal{Q}}$ also shows that irrespective of the choice of $\mathcal{Q}$, on average, random pure states from the $\mathrm{W}$-class are more nonmonogamous than that from the GHZ-class.

Remark: Since the $\alpha_{c}$-value is very large for work deficit (>10), $\mathcal{M}_{\mathcal{W}_{\leftarrow}}$ and $\mathcal{M}_{\mathcal{W}_{\rightarrow}}$ are not numerically discernible in this case.

Note: In the remainder of the paper, whenever we refer to quantum discord or work deficit, the measurement will be performed in the second party.

\begin{tabular}{|c|c|c|}
\hline Measure & Class & $\mathcal{M}_{\mathcal{Q}}$ \\
\hline \multirow{2}{*}{ Negativity $(\mathcal{N})$} & GHZ & 0.7245 \\
\cline { 2 - 3 } & $\mathrm{W}$ & 0.9441 \\
\hline \multirow{2}{*}{ Logarithmic negativity $(\mathcal{L})$} & $\mathrm{GHZ}$ & 0.8765 \\
\cline { 2 - 3 } & $\mathrm{W}$ & 1.0957 \\
\hline \multirow{2}{*}{ Concurrence $(\mathcal{C})$} & $\mathrm{GHZ}$ & 0.9498 \\
\cline { 2 - 3 } & $\mathrm{W}$ & 2.0000 \\
\hline \multirow{2}{*}{ Entanglement of formation $\left(E_{o} F\right)$} & $\mathrm{GHZ}$ & 0.6182 \\
\cline { 2 - 3 } & $\mathrm{W}$ & 1.2751 \\
\hline \multirow{2}{*}{ Quantum discord (left) $\left(\mathcal{D}_{\rightarrow}\right)$} & $\mathrm{GHZ}$ & 0.6669 \\
\cline { 2 - 3 } & $\mathrm{W}$ & 1.4870 \\
\hline \multirow{2}{*}{ Quantum discord (right) $\left(\mathcal{D}_{\leftarrow}\right)$} & $\mathrm{GHZ}$ & 0.6472 \\
\cline { 2 - 3 } & $\mathrm{W}$ & 1.2333 \\
\hline
\end{tabular}

TABLE II: $\mathcal{M}_{\mathcal{Q}}$ for various quantum correlation measures, $\mathcal{Q}$, for three-qubit pure states. 

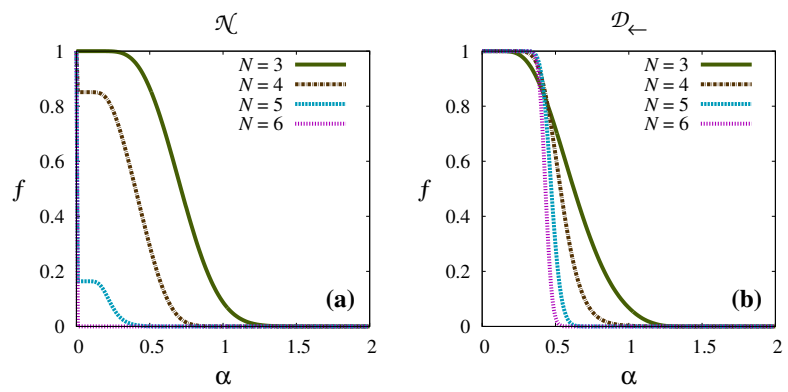

FIG. 3: (Color online.) Plot of $f$ against $\alpha$ with different number of parties, $N$. In (a), negativity is chosen as an entanglement measure, while in (b), $\mathcal{D}_{\leftarrow}$ is plotted. Comparing (a) and (b), we note that unlike entanglement measures, $f$ for information-theoretic measures do not show any jump with $\alpha \rightarrow 0+$. All axes are dimensionless.

\section{B. Uniformity in criticalities for multiqubit states}

Let us now move on to the computation of criticalities in $\alpha$ for random pure states of higher number of qubits. Specifically, we focus on the change in the value of $\alpha_{c}$ for a given $\mathcal{Q}$ with increasing number of parties, $N$. We observe the emergence of a universal feature of $\alpha_{c}$ with respect to $\mathcal{Q}$ when the number of qubits is varied. However, there is a stark contrast in the response of $f$ and $\alpha_{p}$ obtained for entanglement and information-theoretic measures with the increase in the number of parties. Let us now enumerate the similarities and differences of $\delta_{\mathcal{Q}^{\alpha}}$ by varying the number of qubit, $N$, from 3 to 6 .

1. The value of $\alpha_{c}$ and $\mathcal{M}_{\mathcal{Q}}$ decreases for all $\mathcal{Q}$ with the increase of $N$. See Fig. 3 for some typical QC measures. Similar traits can also be seen for other QC measures.

2. Although $\alpha_{c}$ shows some similarities, the fraction of nonmonogamous states demonstrates contrasting behavior depending on the choices of the measure. In particular, in case of entanglement measures, $f$ starts from 1 at $\alpha=0$ and then jumps to a value which is strictly smaller than 1 with $\alpha \neq 0$, thereby causing a discontinuity in $f$ with $\alpha$. Such discontinuity in $f$ is not observed in case of information theoretic QC measures.

3. With the increase of $N$, the value of $\alpha_{p}$ for entanglement measures decreases, while it is increasing for information theoretic ones. We observe this feature for moderate number of parties.

The above observations indicate that for moderate number of qubits, all the random pure states become monogamous with respect to arbitrary QC measures. We will analyze this in greater detail in the succeeding sections and examine the transition from nonmonogamy to monogamy with the variation of the number of parties.

\section{STATISTICS OF MONOGAMY SCORES FOR MULTIPARTITE RANDOM PURE STATES}

In recent years, it has been argued, by using the concept of geometry of quantum states [48], that random pure multipartite states with a moderate number of parties are highly entangled [39, 40, 49, 50], and hence they can be resourceful in quantum information processing tasks, especially in quantum communication protocols. In this section, we investigate the distribution of monogamy scores of random pure multiqubit states for different QC measures. The patterns of distribution of monogamy scores show some universal characteristics irrespective of the measures used to probe them. We first report the difference between the distribution patterns of the GHZ- and the W-class states. We then move on to a higher number of parties and comment how the corresponding spreads change when we consider random pure states with more than three parties. The results obtained in this regard, is in good agreement with the previous results based on geometric entanglement measures.

\section{A. Distribution of monogamy scores for multipartite QC measures of three-party states: GHZ vs. W class states}

To carry out the investigation of three-qubit pure states, we generate $10^{6}$ pure states $|\psi\rangle_{A B C}$, Haar uniformly from both the GHZ- and the W-classes (see Appendix A). The analysis is performed by considering the entanglement and information-theoretic measures as well as their squares. We are interested to scrutinize the qualitative characteristics of monogamy scores for $\mathcal{Q}^{\alpha}$, i.e. $\delta_{\mathcal{Q}}^{\alpha}$ in $\alpha<\alpha_{c}$ and $\alpha>\alpha_{c}$ regions by considering certain statistical quantities, viz. mean, variance, and skewness [51] defined as

$$
\begin{aligned}
\text { Mean: } \mu & =\left\langle\delta_{\mathcal{Q}^{\alpha}}\right\rangle \\
\text { Variance: } \sigma^{2} & =\left\langle\delta_{\mathcal{Q}^{\alpha}}^{2}\right\rangle-\mu^{2} \\
\text { Skewness: } \kappa & =\left\langle\left(\frac{\delta_{\mathcal{Q}^{\alpha}}-\mu}{\sigma}\right)^{3}\right\rangle .
\end{aligned}
$$

The key traits that emerge from the inspection of the GHZ- and the W-class states are summarized below:

1. The first important feature, we notice, is the universality in the distribution of monogamy scores for various QC measures in case of random pure states, belonging to the GHZ-class. In particular, we find that the mean of the distribution of $\delta_{\mathcal{Q}}$ are always positive for all $\mathcal{Q}$, as well as it is positively skewed leading to the Bell typed distribution tilted towards zero. Quantitatively, the $\sigma_{\delta_{\mathcal{Q}}}^{2}$ and $\kappa_{\delta_{\mathcal{Q}}}$ values tend to lie within small windows, viz. $0.02<\sigma_{\delta_{\mathcal{Q}}}^{2}<0.05$ and $0.48<\kappa_{\delta_{\mathcal{Q}}}<0.65$. See Table. III.

2. On the other hand, random pure states chosen from the W-class do not show any qualitatively similar 

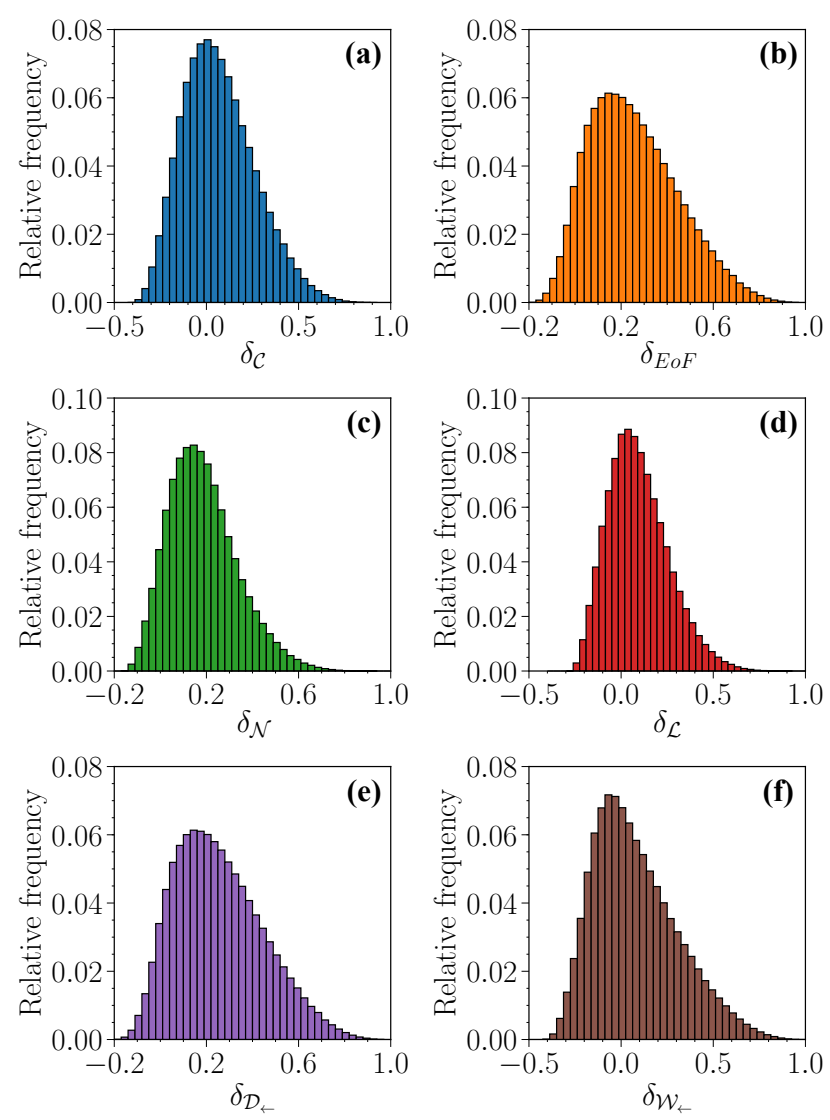

FIG. 4: (Color online.) Relative frequency of monogamy scores for random three-qubit states. Measures chosen are (a) concurrence (b) entanglement of formation (c) negativity (d) logarithmic negativity (e) quantum discord (right) and (f) quantum work deficit (right). Note that Haar uniform simulation of three-qubit random pure states always belong to the GHZ-class. The shape of the distribution remains qualitatively similar if one considers $\delta_{\mathcal{Q}^{2}}$ as also seen from Table. IV. All axes are dimensionless.

pattern. For example, we observe that mean and skewness of $\delta_{\mathcal{Q}}^{\mathrm{W}}$ do not even have the same sign for different QC measures (see Table. III and Fig. 5).

3. Furthermore, our analysis reveal that for the GHZclass states, distributions of $\delta_{\mathcal{Q}^{2}}^{\mathrm{GHZ}}$ and $\delta_{\mathcal{Q}}^{\mathrm{GHZ}}$ are qualitatively similar (see Tables. III and IV). However, for W-class states, a contrasting behavior in the distributions of $\delta_{\mathcal{Q}}^{\mathrm{W}}$ and $\delta_{\mathcal{Q}^{2}}^{\mathrm{W}}$ are observed. See Fig. 5.

As it is already known from [46], an easy test to distinguish two inequivalent classes in three-qubit pure states is to find the tangle $\left(\delta_{\mathcal{C}^{2}}\right)$. Vanishing tangle guarantees the $\mathrm{W}$-class states while the non vanishing value of $\delta_{\mathcal{C}^{2}}$ ensures the GHZ-class states. The above analysis provides a course-grained picture of the properties that are typical to all the QC measures, and are not just restricted to any specific one.

\begin{tabular}{|c|c|c|c|c|}
\hline $\mathcal{Q}$ & Class & Mean & Variance & Skewness \\
\hline \multirow{2}{*}{$\mathcal{N}$} & GHZ & 0.18542 & 0.022174 & 0.62577 \\
\cline { 2 - 5 } & $\mathrm{W}$ & 0.025438 & 0.0069282 & 0.38597 \\
\hline \multirow{2}{*}{$\mathcal{L}$} & $\mathrm{GHZ}$ & 0.094092 & 0.026725 & 0.59757 \\
\cline { 2 - 5 } & $\mathrm{W}$ & -0.023887 & 0.01132 & 0.15495 \\
\hline \multirow{2}{*}{$\mathcal{C}$} & $\mathrm{GHZ}$ & 0.068952 & 0.037962 & 0.48944 \\
\cline { 2 - 5 } & $\mathrm{W}$ & -0.19631 & 0.0089384 & -0.076814 \\
\hline \multirow{2}{*}{ EoF } & $\mathrm{GHZ}$ & 0.25496 & 0.036393 & 0.50209 \\
\cline { 2 - 5 } & $\mathrm{W}$ & -0.062687 & 0.0022365 & -0.66105 \\
\hline \multirow{2}{*}{$\mathcal{D}_{\leftarrow}$} & GHZ & 0.25496 & 0.036393 & 0.50209 \\
\cline { 2 - 5 } & $\mathrm{W}$ & -0.062687 & 0.0022365 & -0.66105 \\
\hline \multirow{2}{*}{$\mathcal{W}_{\leftarrow}$} & GHZ & 0.079392 & 0.051408 & 0.64816 \\
\cline { 2 - 5 } & $\mathrm{W}$ & -0.085636 & 0.0033781 & -0.9464 \\
\hline
\end{tabular}

TABLE III: Mean, variance and skewness values for the GHZand the $\mathrm{W}$-class states for various QC measures.

\begin{tabular}{|c|c|c|c|c|}
\hline $\mathcal{Q}^{2}$ & Class & Mean & Variance & Skewness \\
\hline \multirow{2}{*}{$\mathcal{N}^{2}$} & GHZ & 0.413269 & 0.027109 & 0.19832 \\
\cline { 2 - 5 } & $\mathrm{W}$ & 0.14462 & 0.015031 & 0.90635 \\
\hline \multirow{2}{*}{$\mathcal{L}^{2}$} & $\mathrm{GHZ}$ & 0.37581 & 0.023849 & 0.40478 \\
\cline { 2 - 5 } & $\mathrm{W}$ & 0.13071 & 0.010451 & 0.76232 \\
\hline \multirow{2}{*}{$\mathcal{C}^{2}$} & $\mathrm{GHZ}$ & 0.33335 & 0.034293 & 0.50371 \\
\cline { 2 - 5 } & $\mathrm{W}$ & 0 & - & - \\
\hline \multirow{2}{*}{$E F^{2}$} & $\mathrm{GHZ}$ & 0.3985 & 0.040395 & 0.32512 \\
\cline { 2 - 5 } & $\mathrm{W}$ & 0.060853 & 0.0036504 & 1.1312 \\
\hline \multirow{2}{*}{$\mathcal{D}_{\leftarrow}^{2}$} & $\mathrm{GHZ}$ & 0.42308 & 0.040715 & 0.23827 \\
\cline { 2 - 5 } & $\mathrm{W}$ & 0.074828 & 0.0043115 & 0.84245 \\
\hline \multirow{2}{*}{$\mathcal{W}_{\leftarrow}^{2}$} & $\mathrm{GHZ}$ & 0.31472 & 0.043941 & 0.46134 \\
\cline { 2 - 5 } & $\mathrm{W}$ & 0.058729 & 0.003126 & 0.70358 \\
\hline
\end{tabular}

TABLE IV: Mean, variance and skewness values for the GHZand the $\mathrm{W}$-class states for the squares of various QC measures.

\section{B. Monogamy scores mimic properties of multipartite entanglement measures}

In the preceeding section, we observe that all the distribution of monogamy scores for random three-qubit pure states which typically belong to the GHZ-class, follow a similar pattern. Such behavior is nicely reflected in the values of skewness, which turns out to be around 0.5 for all the QC measures. Since previous studies show that random $N$-party pure states, with $N>11$ are highly entangled $[39,40]$, it will be interesting to see the distribution of the monogamy scores for higher number of parties. In this respect, let us first consider the monogamy score in an asymptotic scenario.

Theorem 1. For a random pure state of $N$ qubits, the monogamy score for any entanglement measure $\mathcal{Q}$ approaches to unity with the increase of the number of parties provided the entanglement measure reduces to von 

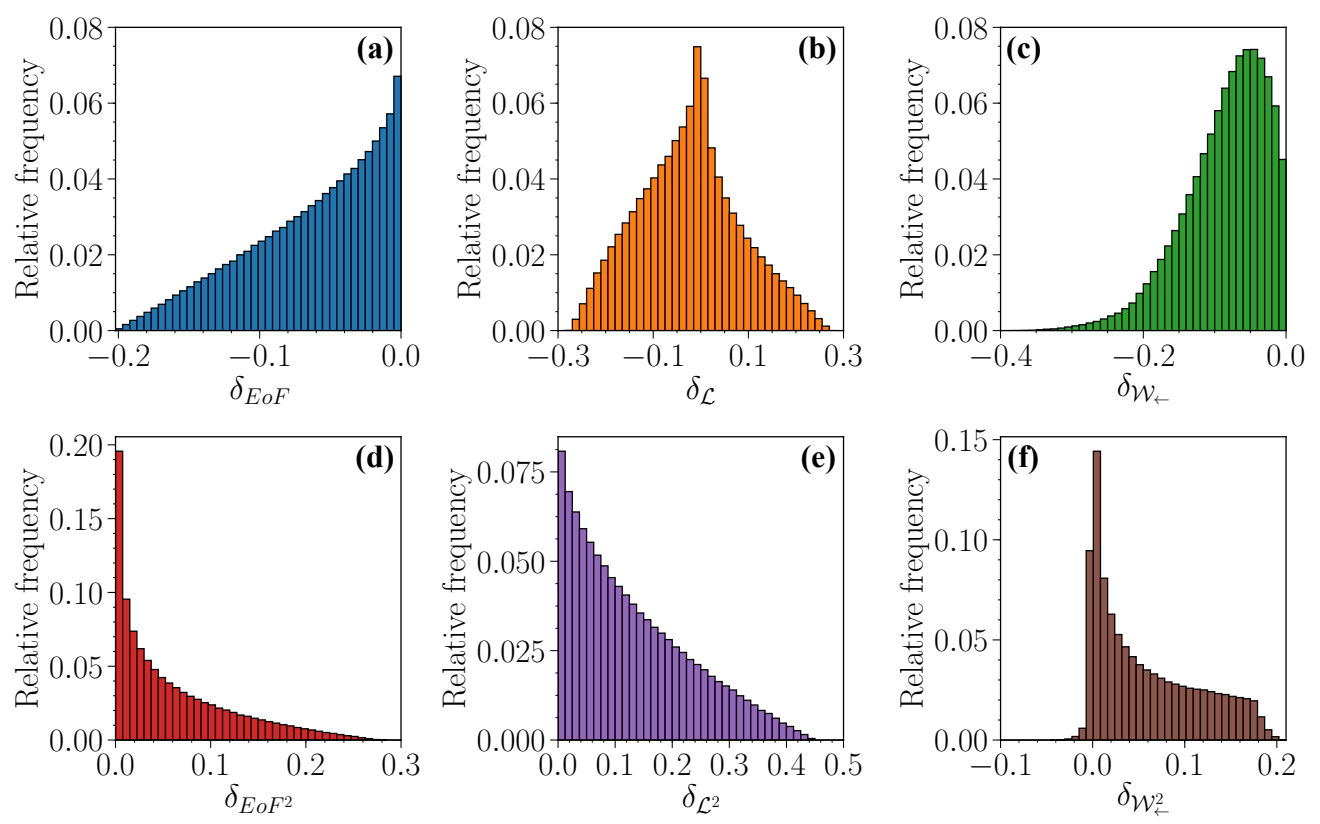

FIG. 5: (Color online.) Relative frequency of $\delta_{\mathcal{Q}}$ of states from the W-class (a) $E$ oF (b) $\mathcal{L}$ and (c) $\mathcal{W}_{\leftarrow}$. In (d)-(f), similar measures are chosen for studying the distribution of $\delta_{\mathcal{Q}^{2}}$. Unlike the GHZ-class, the distribution of $\delta_{\mathcal{Q}}$ are quite contrasting to $\delta_{\mathcal{Q}^{2}}$ (see Tables. III and IV). All axes are dimensionless.
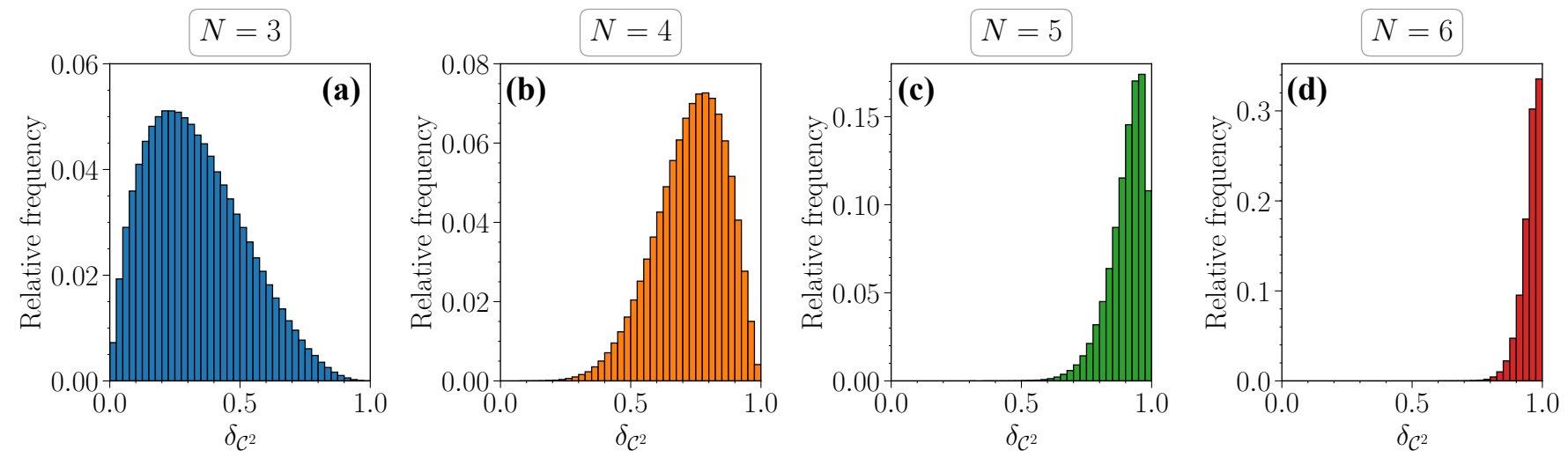

FIG. 6: (Color online.) Patterns of distribution of monogamy scores for squared concurrence for randomly chosen pure states with (a) three-, (b) four-, (c)five- and (d) six-qubits. Figure (d) confirms that most of the random six-qubit pure states possess high values of $\delta_{\mathcal{C}^{2}}$. All axes are dimensionless.

Neumann entropy of the local density matrix for pure states.

Proof. For pure states, of $N$ parties, $|\psi\rangle_{123 \ldots N}$, the first term in the monogamy score defined in Eq. (1), $\mathcal{Q}_{1 \text { :rest }}$, for a large variety of entanglement measures, reduces to the von Neumann entropy of the reduced density matrix, $S\left(\rho_{1}\right)=-\operatorname{tr}\left(\rho_{1} \log _{2} \rho_{1}\right)$, where $\rho_{1}=$ $\operatorname{tr}_{23 \ldots N}\left(|\psi\rangle_{123 \ldots N}\langle\psi|\right)$. For a state with $N$ qubits, the mean entropy, averaged over the Haar measure, reads
$[52-54]$

$$
\left\langle S\left(\rho_{1}\right)\right\rangle=\log _{2} e\left(\sum_{2^{N-1}+1}^{2^{N}} \frac{1}{j}-\frac{1}{2^{N}}\right) \approx 1-\frac{\log _{2} e}{2^{N-1}},
$$

so that for $N \rightarrow \infty$, we have

$$
\left\langle\mathcal{Q}_{1 \text { rest }}\right\rangle=\left\langle S\left(\rho_{1}\right)\right\rangle_{N \rightarrow \infty} \rightarrow 1 .
$$

Although we have taken the first party to be the nodal observer, the results remain unchanged for other nodal observers as well. Therefore almost all states, $\mathcal{Q}_{1 \text { :rest }}=1$ for large $N$. 

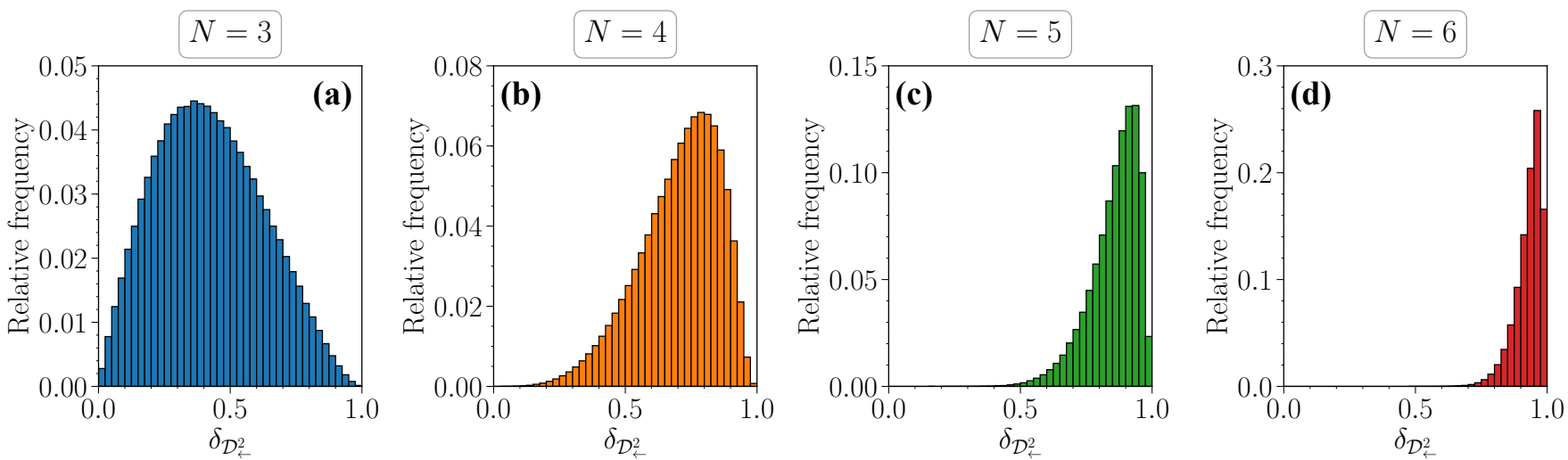

FIG. 7: (Color online.) Trends of distribution of $\delta_{\mathcal{D}_{\leftarrow}^{2}}$ for different values of $N\left((\mathrm{a})\right.$-(d) $3,4,5$ and 6 qubits). Note that $\delta_{\mathcal{C}^{2}}$ and $\delta_{\mathcal{D}_{\leftarrow}^{2}}$ behave in a similar fashion and their behavior is same as multiparty geometric entanglement measures as reported in $[40,50]$. All axes are dimensionless.

Let us now try to put bounds on the bipartite $\mathrm{QC}, Q_{1: i}$ in Eq. (1). If we restrict ourselves to spin-1/2 particles, all $\rho_{1: i}$ are two-qubit states having rank 2. Moreover, we know $[7,8]$ that the partial transposition criterion is necessary and sufficient for entanglement in this case. All bipartite states obtained from an $N$ qubit random pure state will have positive partial transpose [49] for $N>6+\log _{2} \frac{11}{16}$, and hence are separable, having vanishing entanglement, i.e. we get $\mathcal{Q}_{1: i}$ identically vanishing for sufficiently large $N$, for almost all states. Therefore, for the entanglement measures considered, for sufficiently large values of $N$,

$$
\delta_{\mathcal{Q}}=1,
$$

for almost all $N$-qubit states. This completes the proof.

Remark: Numerical simulations of four, five and sixqubit states unveils that $\left\langle\mathcal{Q}_{1: i}\right\rangle$ approaches its extremal value, i.e., $\left\langle\mathcal{Q}_{1: i}\right\rangle \rightarrow 0$, faster than the way $\left\langle\mathcal{Q}_{1 \text { rest }}\right\rangle$ approaches unity with the increase of $N$.

Numerical simulations reveal that not only for entanglement measures, but also for information-theoretic QC measures, for high $N$, say for random six-qubit states, $\mathcal{Q}\left(\rho_{1: i}\right) \rightarrow 0$ and $\left\langle\delta_{\mathcal{Q}}\right\rangle \rightarrow 1$ as $\left\langle\mathcal{Q}_{1 \text { :rest }}\right\rangle \rightarrow 1$, which is in agreement with the asymptotic case. Let us now describe the way by which it approaches to unity for random multiparty pure states (cf. [40]).

We again Haar uniformly [48] generate random pure states of three-, four-, five- and six-qubits and compute $\delta_{\mathcal{Q}^{2}}$. To analyze the data in a quantitative manner, we evaluate mean, variance and skewness for various measures as presented in Table. V. Figs. 6 and 7 highlight the change in the distribution of $\delta_{\mathcal{C}^{2}}$ and $\delta_{\mathcal{D}_{\leftarrow}^{2}}$ with the variation of number of qubits. Analysis of the Tables $\mathrm{V}$ and VI, and Figs. 6 and 7 reveal certain aspects of monogamy scores -

1. Means of $\delta_{\mathcal{Q}^{2}}$ increases with $N \forall \mathcal{Q}$ and becomes more than 0.92 for $N=6$. Moreover, entanglement measures approach unity faster than the information theoretic one.

2. All the QC measures make a transition from being right skewed to being skewed to the left with $N \geq 4$.

Theorem 1 coupled with the numerical simulations gives a comprehensive picture of how the distributions of monogamy score approaches unit mean with the increase of $N$. Therefore, almost all the random pure states not only satisfy monogamy relation for any arbitrary squared QC measure, $\mathcal{Q}^{2}$, but it possess a very high value of the same with states having moderate number of parties. For example, among $10^{6}$ random six-qubit pure states, $91.34 \%$ states have $\delta_{\mathcal{C}^{2}}$ more than 0.9 .

The geometric measure of entanglement [55], defined as $E_{g}(|\psi\rangle)=-\log _{2} \sup _{\alpha \in \mathcal{P}}|\langle\alpha \mid \psi\rangle|^{2}$ with $\mathcal{P}$ being the set of all product states, rises rapidly with the increase in the number of parties. Specifically, it was shown that the fraction of states having $E_{g} \leq N-2 \log _{2}(N)-3$ is smaller than $e^{-N^{2}}$. Our results which shows how the maximal value is attained by increasing the number of parties have a striking resemblance with the above result. Moreover, this similarity is independent of the measure used to construct the monogamy scores. Thus, we can safely assert that on increasing the number of parties, almost all randomly chosen pure states have maximal monogamy score and that the state possess high QC. 


\begin{tabular}{|c|c|c|c|c|c|c|}
\hline \multicolumn{2}{|c|}{$X=\mu, \sigma^{2}, \kappa$} & \multicolumn{2}{|c|}{ Mean } & \multicolumn{2}{c|}{ Variance } & \multicolumn{2}{c|}{ Skewness } \\
\cline { 1 - 7 } $\mathcal{Q}^{2} \downarrow$ & 3 & 6 & 3 & 6 & 3 & 6 \\
\hline $\mathcal{C}^{2}$ & 0.33335 & 0.95732 & 0.034293 & 0.0013211 & 0.50371 & -1.4482 \\
\hline $\mathcal{N}^{2}$ & 0.41329 & 0.95373 & 0.027109 & 0.0013209 & 0.19832 & -1.4483 \\
\hline$E o F^{2}$ & 0.3985 & 0.93432 & 0.040395 & 0.00259 & 0.32512 & -1.3846 \\
\hline $\mathcal{L}^{2}$ & 0.37581 & 0.96605 & 0.023849 & 0.00073 & 0.040478 & -1.5107 \\
\hline $\mathcal{D}_{\leftarrow}^{2}$ & 0.42308 & 0.92927 & 0.040715 & 0.0025693 & 0.23827 & -1.3762 \\
\hline
\end{tabular}

TABLE V: Statistical quantities $\left(X=\mu, \sigma^{2}, \kappa\right)$ of $\delta_{\mathcal{Q}^{2}}$ for three- and six-qubit states.

\begin{tabular}{|c|c|c|c|c|}
\hline$X=\mu$ & \multicolumn{4}{|c|}{ Mean } \\
\hline $\mathcal{Q}^{2} \downarrow \rightarrow$ & 3 & 4 & 5 & 6 \\
\hline $\mathcal{C}^{2}$ & 0.33335 & 0.72941 & 0.90175 & 0.95732 \\
\hline $\mathcal{N}^{2}$ & 0.41329 & 0.75322 & 0.90303 & 0.95373 \\
\hline$E o F^{2}$ & 0.3985 & 0.73239 & 0.87166 & 0.93432 \\
\hline $\mathcal{L}^{2}$ & 0.37581 & 0.75106 & 0.92158 & 0.96605 \\
\hline $\mathcal{D}_{\leftarrow}^{2}$ & 0.42308 & 0.70413 & 0.85582 & 0.92927 \\
\hline
\end{tabular}

TABLE VI: Mean of $\delta_{\mathcal{Q}^{2}}$ for $N=3$ to 6 parties.

Furthermore, it was shown [46] that monogamy scores with squared concurrence, $\delta_{\mathcal{C}^{2}}$, also known as three tangle is an entanglement monotone, and consequently all its positive powers are also valid entanglement measures. Although this is no longer true for monogamy scores based on other QC measures. Analyzing our data, we can conclude that if the monogamy scores are properly written, so that non-negativity is guaranteed, then their distributions mimic the pattern of multiparty entanglement for moderate number of parties. Moreover, our results also establish a connection between the restrictions in sharability of bipartitie QC in a multiparty quantum state and the amount of multiparty QC contained in the state.

\section{CONCLUSION}

For multiparty quantum states, the knowledge about the distribution of quantum correlation among its various parties lies at the heart of the characterization of multiparty entanglement or more generally, multiparty quantum correlations (QC). Unlike classical correlations, quantum mechanics puts restriction on arbitrary sharing of QC. Such constraints are encapsulated in the concept of monogamy and is quantified in terms of the monogamy score.

The monogamy score can be defined for any valid quantum correlation measure and the same raised to any nonnegative power. In this paper, we compute the critical monogamy power above which all the random pure states becomes monogamous for several paradigmatic QC measures. We also show that it decreases with the increase in number of parties. We find that the fraction of nonmonogamous states remains frozen for certain powers of QC measure in a monogamy score.

Both analytically and numerically, we report that the mean of the distribution of monogamy scores for large number of parties of random pure multiqubit states approaches to unity irrespective of the QC measure. Our analysis unveils an universal character, independent of the measure, in the distribution of monogamy scores and its statistically relevent quantities like mean, variance and skewness.

From the distribution of monogamy score, it is clear that almost all random pure states having moderate number of parties are not only monogamous with respect to any QC measure, but possess high value of the corresponding monogamy score. Such results can have some important applications towards building quantum cryptography involving multiple parties. Moreover, the observations in behavior of monogamy scores are in good agreement with the known results obtained for geometric measure of entanglement for random multiparty states. Additionally, our analysis of mean and skewness of the distributions show the path in which the distributions change with the variation of the number of parties.

\section{Acknowledgments}

This research was supported in part by the 'INFOSYS scholarship for senior students'. Numerical results have been obtained using the Quantum Information and Computation library (QIClib) (https://titaschanda. github.io/QIClib). TC was supported in part by Quantera QTFLAG project 2017/25/Z/ST2/03029 of National Science Centre (Poland). Soorya Rethinasamy acknowledges the hospitality of Harish-Chandra Research Institute.

\section{Appendix A}

We describe the parameterizations used to describe the two SLOCC inequivalent classes of three-qubit pure states. Furthermore, we provide definitions of the quantum correlation measures, used in the main text. 


\section{GHZ- and W-class states}

Any arbitrary three-qubit random pure state can be represented as

$$
|\psi\rangle=\sum_{i=1}^{8} \alpha_{i}\left|i_{1} i_{2} i_{3}\right\rangle
$$

where $\alpha_{i}=\alpha_{i}^{\prime}+i \alpha_{i}^{\prime \prime}$, with $\alpha_{i}^{\prime}$ and $\alpha_{i}^{\prime \prime}$ being chosen randomly from a normal distribution with mean 0 and variance unity and $\left|i_{k}\right\rangle=|0\rangle$ or $|1\rangle, k=1,2,3$. Any random pure three-qubit state belongs to the GHZ-class, having $\delta_{\mathcal{C}^{2}}>0$

On the other hand, another important class of threequbit pure states which are SLOCC-inequivalent with the GHZ-class is the W-class states, given by

$$
\left|\psi_{W}\right\rangle=\sqrt{a}|001\rangle+\sqrt{b}|010\rangle+\sqrt{c}|100\rangle+\sqrt{d}|000\rangle
$$

upto local unitary operations. Since among three-qubit pure states, the $\mathrm{W}$-class states belong to the set of measure zero, simulation of random three-qubit pure states do not produce states from the W-class. Since it is an important class and it's trends of the distribution can be important, we numerically simulate the W-class states by randomly choosing parameters from normal distribution.

\section{Entanglement Measures}

Some typical measures from the entanglement separability paradigm are discussed below. For a pure bipartite quantum state, $\left|\psi_{A B}\right\rangle$, entanglement is uniquely defined as the von Neumann entropy of its reduced density matrices, given by

$$
\mathcal{E}\left(\left|\psi_{A B}\right\rangle\right)=\mathcal{S}\left(\rho_{A}\right)=\mathcal{S}\left(\rho_{B}\right)
$$

where $\mathcal{S}(\sigma) \equiv-\operatorname{tr}(\sigma \log \sigma)$, and $\rho_{A}$ and $\rho_{B}$ are the local density matrices of $\left|\psi_{A B}\right\rangle$. For mixed bipartite states, there are more than one inequivalent quantifiers of entanglemnt. The ones considered in this manuscript are the known computable ones.

1. The entanglement of formation [13] for a mixed state $\rho_{A B}$ is defined as

$$
\operatorname{EoF}\left(\rho_{A B}\right)=\min _{\left\{p_{i},\left|\psi_{A B}^{i}\right\rangle\right\}} \sum_{i} p_{i} \mathcal{E}\left(\left|\psi_{A B}^{i}\right\rangle\right)
$$

where the minimization is taken over all possible pure state decompositions, $\rho_{A B}=\sum_{i} p_{i}\left|\psi_{A B}^{i}\right\rangle\left\langle\psi_{A B}^{i}\right|$. It can be computed for arbitrary two-qubit states, explained below.

2. The concurrence [14] for a pure state, $\left|\psi_{A B}\right\rangle$, is defined as

$$
\mathcal{C}\left(\psi_{A B}\right)=\left|\left\langle\psi_{A B} \mid \tilde{\psi}_{A B}\right\rangle\right|
$$

where $\left|\tilde{\psi}_{A B}\right\rangle=\sigma_{y} \otimes \sigma_{y}\left|\psi_{A B}^{*}\right\rangle$ with $\left|\psi_{A B}^{*}\right\rangle$ being the complex conjugate of $\left|\psi_{A B}\right\rangle$ in the computational basis $\{|00\rangle,|01\rangle,|10\rangle,|11\rangle\}$.

For a general two-qubit density matrix, $\rho_{A B}$, we first define the spin flipped density matrix, $\tilde{\rho}_{A B}=$ $\left(\sigma_{y} \otimes \sigma_{y}\right) \rho_{A B}^{*}\left(\sigma_{y} \otimes \sigma_{y}\right)$, and the operator $\mathcal{R}=$ $\sqrt{\sqrt{\rho_{A B}} \rho_{A B}^{*} \sqrt{\rho_{A B}}}$. The definition of concurrence then reduces to $\mathcal{C}\left(\rho_{A B}\right)=\min \left\{0, \lambda_{1}-\lambda_{2}-\lambda_{3}-\lambda_{4}\right\}$, where $\lambda_{i}$ s are the eigenvalues of $R$ in decreasing order. The entanglement of formation is then given by $\operatorname{EoF}\left(\rho_{A B}\right)=\mathcal{F}\left(\mathcal{C}\left(\rho_{A B}\right)\right)$ where $\mathcal{F}(\mathcal{C})$ reads as

$$
\mathcal{F}(\mathcal{C})=h\left(\frac{1+\sqrt{1-\mathcal{C}^{2}}}{2}\right),
$$

with $h(x)=-x \log _{2}(x)-(1-x) \log _{2}(1-x)$ being the well known binary entropy.

3. Negativity [15] is defined as the twice of the absolute sum of negative eigenvalues of the partially transposed density matrix, $\rho_{A B}^{T_{B}}$, where partial transposition is taken with respect to $B$. Mathematically,

$$
\mathcal{N}\left(\rho_{A B}\right)=\left\|\rho_{A B}^{T_{B}}\right\|-1=\left\|\rho_{A B}^{T_{A}}\right\|-1,
$$

where $\|A\|=\operatorname{tr} \sqrt{A^{\dagger} A}$.

Note that we multiply by 2 to make its maximum for two-qubit maximally entangled states to be unity.

4. The logarithmic negativity $[15,16]$ for a bipartite state $\rho_{A B}$ is defined in terms of negativity as

$$
\mathcal{L}\left(\rho_{A B}\right)=\log _{2}\left(\mathcal{N}\left(\rho_{A B}\right)+1\right)
$$

positivity of $\mathcal{L}\left(\rho_{A B}\right)$ guarantees that the state is entangled and distillable.

\section{QC Measures Independent of Entanglement}

Some representative quantum correlation measures from the information-theoretic paradigm are given below.

\section{Quantum discord:}

Uninterrogated quantum conditional entropy of a bipartite state $\rho_{A B}$, shared between $A$ and $B$, is defined as $\tilde{\mathcal{S}}\left(\rho_{A \mid B}\right)=\mathcal{S}\left(\rho_{A B}\right)-\mathcal{S}\left(\rho_{B}\right)$. Similarly, the interrogated conditional entropy is defined as

$$
\mathcal{S}\left(\rho_{A \mid B}\right)=\min _{\Pi_{i}^{B}} \sum_{i} p_{i} \mathcal{S}\left(\rho_{A \mid i}\right)
$$

where the minimization is performed over all complete sets of projective measurements with

$$
\rho_{A \mid i}=\operatorname{Tr}_{B}\left[\left(\mathbb{I}_{A} \otimes \Pi_{i}^{B}\right) \rho_{A B}\left(\mathbb{I}_{A} \otimes \Pi_{i}^{B}\right)\right] / p_{i},
$$


where $p_{i}=\operatorname{Tr}_{A B}\left[\left(\mathbb{I}_{A} \otimes \Pi_{i}^{B}\right) \rho_{A B}\left(\mathbb{I}_{A} \otimes \Pi_{i}^{B}\right)\right]$.

Hence, uninterrogated quantum mutual information can be written as

$$
\tilde{I}\left(\rho_{A B}\right)=\mathcal{S}\left(\rho_{A}\right)-\tilde{\mathcal{S}}\left(\rho_{A \mid B}\right),
$$

while the interrogated quantum mutual information reads as

$$
I_{\leftarrow}\left(\rho_{A B}\right)=\mathcal{S}\left(\rho_{A}\right)-\mathcal{S}\left(\rho_{A \mid B}\right),
$$

where the symbol " $\leftarrow$ " in the subscript indicates that the measurement is performed on the second subsystem, B.

Quantum discord $[17,18]$ is defined as the difference between uninterrogated and interrogated quantum mutual information, given by

$$
\mathcal{D}_{\leftarrow}\left(\rho_{A B}\right)=\tilde{I}\left(\rho_{A B}\right)-I_{\leftarrow}\left(\rho_{A B}\right),
$$

which can be called quantum discord (right). Naturally, when measurement is performed in the first party, we get quantum discord (left), denoted by $\mathcal{D}_{\rightarrow}$. Throughout the paper, unless mentioned, we compute $\mathcal{D}_{\leftarrow}$. The results remain qualitatively unchanged for $\mathcal{D}_{\rightarrow}$.

\section{Quantum work deficit:}

The amount of extractable pure states under a set of global operations, called "closed operations" $(\mathrm{CO})$ is given by

$$
I_{C O}=\log _{2} \operatorname{dim}(\mathcal{H})-\mathcal{S}\left(\rho_{A B}\right) .
$$

On the other hand, the amount of extractable pure states under a set of closed local operations and classical communication (CLOCC) can be

$$
I_{C L O C C}=\log _{2} \operatorname{dim}(\mathcal{H})-\min \mathcal{S}\left(\rho_{A B}^{\prime}\right)
$$

with minimizaion being performed over all local projective measurements on the second party and where $\rho_{A B}^{\prime}=\sum p_{i} \rho_{A \mid i}$. Quantum work deficit $[20,21]$ can be argued as a measure of QC and is defined to the residual work extraction by $\mathrm{CO}$ after extraction by CLOCC and can be represented as

$$
\mathcal{W}_{\leftarrow}\left(\rho_{A B}\right)=I_{C O}-I_{C L O C C} .
$$

Like quantum discord, when the measurement is performed in the first party, we have $\mathcal{W}_{\rightarrow}$.
[1] R. Horodecki, P. Horodecki, M. Horodecki, and K. Horodecki, Rev. Mod. Phys. 81, 865 (2009), and references therein.

[2] C. H. Bennett, Phys. Scr. 1998, 210 (1998).

[3] C. H. Bennett, G. Brassard, C. Crépeau, R. Jozsa, A. Peres, and W. K. Wootters, Phys. Rev. Lett. 70, 1895 (1993).

[4] C. H. Bennett and S. J. Wiesner, Phys. Rev. Lett. 69, 2881 (1992).

[5] A. K. Ekert, Phys. Rev. Lett. 67, 661 (1991).

[6] P. Walther, K. J. Resch, T. Rudolph, E. Schenck, H. Weinfurter, V. Vedral, M. Aspelmeyer, and A. Zeilinger, Nature 434, 169 (2005).

[7] A. Peres, Phys. Rev. Lett. 77, 1413 (1996).

[8] M. Horodecki, P. Horodecki, and R. Horodecki, Phys. Lett. A 223, 1 (1996).

[9] S.L. Woronowicz, Commun. Math. Phys. 51, 243 (1976); P. Kryszynski and S.L. Woronowicz, Lett. Math. Phys.3, 319 (1979); M.D. Choi, Proc. Sympos. Pure Math. 38, 583 (1982).

[10] B.M. Terhal, Lin. Alg. Appl. 323, 61 (2001).

[11] M. Lewenstein, B. Kraus, J.I. Cirac, and P. Horodecki, Phys. Rev. A 62, 052310 (2000); D. Bruß, J.I. Cirac, P. Horodecki, F. Hulpke, B. Kraus, M. Lewenstein, and A. Sanpera, J. Mod. Opt. 49, 1399 (2002).

[12] C. H. Bennett, G. Brassard, S. Popescu, B. Schumacher, J. A. Smolin, and W. K. Wootters, Phys. Rev. Lett. 76, $722(1996)$.

[13] C. H. Bennett, D. P. DiVincenzo, J. Smolin, and W. K. Woott ers, Phys. Rev. A 54, 3824 (1996).

[14] W. K. Wootters, Phys. Rev. Lett. 80, 2245 (1998).
[15] K. Życzkowski, P. Horodecki, A. Sanpera, and M. Lewenstein, Phys. Rev. A 58, 883 (1998); J. Lee, M. S. Kim, Y. J. Park, and S. Lee, J. Mod. Opt. 47, 2151 (2000); G. Vidal and R. F. Werner, Phys. Rev. A 65, 032314 (2002).

[16] M. B. Plenio, Phys. Rev. Lett. 95, 090503 (2005).

[17] K. Modi, A. Brodutch, H. Cable, T. Paterek, and V. Vedral, Rev. Mod. Phys. 84, 1655 (2012); A. Bera, T. Das, D. Sadhukhan, S. S. Roy, A. Sen(De), and U. Sen, Rep. Prog. Phys. 81, 024001 (2018).

[18] W. H. Zurek, Ann. Phys. Lpz. 9, 855 (2000); H. Ollivier and W. H. Zurek, Phys. Rev. Lett. 88, 017901 (2001); L. Henderson and V. Vedral, J. Phys. A: Math. Gen. 34, 6899 (2001).

[19] B. Dakić, V. Vedral, and Č. Brukner Phys. Rev. Lett. 105, 190502 (2010); S. Luo and S. Fu, Phys. Rev. A 82, $034302(2010)$.

[20] J. Oppenheim, M. Horodecki, P. Horodecki, and R. Horodecki, Phys. Rev. Lett. 89, 180402 (2002); M. Horodecki, K. Horodecki, P. Horodecki, R. Horodecki, J. Oppenheim, A. Sen(De), and U. Sen, Phys. Rev. Lett. 90, 100402 (2003); M. Horodecki, P. Horodecki, R. Horodecki, J. Oppenheim, A. Sen(De), and U. Sen, Phys. Rev. A 71, 062307 (2005).

[21] I. Devetak, Phys. Rev. A 71, 062303 (2005).

[22] A. K. Rajagopal and R. W. Rendell, Phys. Rev. A 66, 022104 (2002); A. R. Usha Devi and A. K. Rajagopal, Phys. Rev. Lett. 100, 140502 (2008); Sudha, A. R. Usha Devi, and A. K. Rajagopal, Phys. Rev. A 85, 012103 (2012).

[23] H. S. Dhar, A. K. Pal, D. Rakshit, A. Sen(De), U. Sen, Lectures on General Quantum Correlations and their Ap- 
plications, Part of the series Quantum Science and Technology, Springer International Publishing (2017), pp 2364; arXiv:1610.01069 [quant-ph].

[24] C. H. Bennett, H. J. Bernstein, S. Popescu, and B. Schumacher, Phys. Rev. A 53, 2046 (1996); M. Koashi and A. Winter, Phys. Rev. A 69, 022309 (2004); G. Adesso, A. Serafini, and F. Illuminati, Phys. Rev. A 73, 032345 (2006); T. Hiroshima, G. Adesso, and F. Illuminati, Phys. Rev. Lett. 98, 050503 (2007); M. Hayashi and L. Chen, Phys. Rev. A 84, 012325 (2011); A. Streltsov, G. Adesso, M. Piani, and D Bruß, Phys. Rev. Lett. 109, 050503 (2012); F. F. Fanchini, M. C. de Oliveira, L. K. Castelano, and M. F. Cornelio, Phys. Rev. A 87, 032317 (2013); Y.-K. Bai, Y.-F. Xu, and Z. D. Wang, Phys. Rev. Lett. 113, 100503 (2014); B. Regula, S. D. Martino, S. Lee, and G. Adesso, Phys. Rev. Lett. 113, 110501 (2014); M. Enriquez, F. Delgado, and K. Życzkowski, arXiv:1809.00642 [quant-ph].

[25] V. Coffman, J. Kundu, and W. K. Wootters, Phys. Rev. A 61, 052306 (2000); T. Osborne and F. Verstraete, Phys. Rev. Lett. 96, 220503 (2006).

[26] K. Salini, R. Prabhu, A. Sen(De), and U. Sen, Ann. Phys. 348, 297 (2014).

[27] A. Kumar and H. S. Dhar, Phys. Rev. A 93, 062337 (2016).

[28] R. Prabhu, A. K. Pati, A. Sen(De), and U. Sen, Phys. Rev. A 85, 040102(R) (2012); A. Kumar, Phys. Lett. A 380, 38 (2016).

[29] S. Roy, T. Das, A. Kumar, A. Sen(De), and U. Sen, Phys. Rev. A 98, 012310 (2018).

[30] C. Lancien, S. Di Martino, M. Huber, M. Piani, G. Adesso, and A. Winter, Phys. Rev. Lett. 117, 060501 (2016).

[31] G. Gour and Y. Guo, Quantum 2, 81 (2018).

[32] M. Pawlowski, Phys. Rev. A 82, 032313 (2010).

[33] X. Yang, K. Wei, H. Ma, S. Sun, H. Liu, Z. Yin, Z. Li, S. Lian, Y. Du, and L. Wu, Phys. Rev. A 93, 052303 (2016).

[34] A. Kumar, S. S. Roy, A. K. Pal, R. Prabhu, A. Sen(De), and U. Sen, Phys. Lett. A 380, 3588 (2016).

[35] X.-S. Ma, B. Dakic, W. Naylor, A. Zeilinger, and P. Walther, Nat. Phys. 7, 399 (2011).
[36] K. Meichanetzidis, J. Eisert, M. Cirio, V. Lahtinen, and J. K. Pachos, Phys. Rev. Lett. 116, 130501 (2016).

[37] A. Chandran, D. Kaszlikowski, A. Sen(De), U. Sen, and V. Vedral, Phys. Rev. Lett. 99, 170502 (2007); H. S. Dhar and A. Sen(De), J. Phys. A 44, 465302 (2011); K. Rama Koteswara Rao, H. Katiyar, T. S. Mahesh, A. Sen (De), U. Sen, and A. Kumar, Phys. Rev. A 88, 022312 (2013).

[38] M. N. Bera, R. Prabhu, A. Sen(De), and U. Sen, Phys. Rev. A 86, 012319 (2012).

[39] P. Hayden, D. W. Leung, and A. Winter, Comm. Math. Phys. 265, 1 (2006).

[40] D. Gross, S. T. Flammia, and J. Eisert, Phys. Rev. Lett. 102, 190501 (2009).

[41] X. Zhu and S. Fei, Phy. Rev. A 90, 024304 (2014).

[42] Y. Luo and Y. Li, Ann. Phys. 362, 511 (2015).

[43] Z. Jin, J. Li, T. Li, and S. Fei, Phys. Rev. A 97, 032336 (2018).

[44] Y. -C. Ou and H. Fan, Phys. Rev. A 75, 062308 (2007); H. He and G. Vidal, Phys. Rev. A 91, 012339 (2015); J. H. Choi and J. S. Kim, Phys. Rev. A 92, 042307 (2015).

[45] Y.-K. Bai, N. Zhang, M.-Y. Ye, Z. D. Wang, Phys. Rev. A 88, 012123 (2013).

[46] W. Dür, G. Vidal, and J. I. Cirac, Phys. Rev. A 62, 062314 (2000).

[47] Other parties of a multipartite state can also serve as nodal observer.

[48] I. Bengtsson and K. Zyczkowski, Geometry of Quantum States: An introduction to Quantum Entanglement (Cambridge University Press, 2006).

[49] V. M. Kendon, K. Życzkowski, and W. J. Munro, Phys. Rev. A 66, 062310 (2002).

[50] M. J. Bremner, C. Mora, and A. Winter, Phys. Rev. Lett. 102, 190502 (2009).

[51] M. G. Bulmer, Principles of Statistics, Dover Publications (1965).

[52] D. N. Page, Phys. Rev. Lett. 71, 1291 (1993).

[53] S. K. Foong and S. Kanno, Phys. Rev. Lett. 72, 1148 (1994).

[54] S. Sen, Phys. Rev. Lett. 77, 1 (1996).

[55] A. Shimony, Ann. N.Y. Adad. Sci. 755, 675 (1995); H. Barnum and N. Linden, J. Phys. A 34, 6787 (2001). 